\title{
Discrepancy of Sums of Three Arithmetic Progressions
}

\author{
Aleš Přívětivý * \\ Department of Applied Mathematics of Charles University \\ Malostranské nám. 25, 11800 Praha, Czech Republic \\ privetivy@kam.mff.cuni.cz
}

Submitted: Jul 24, 2005; Accepted: Dec 5, 2005; Published: Jan 25, 2006

Mathematics Subject Classification: 11K38

\begin{abstract}
The set system of all arithmetic progressions on $[n]$ is known to have a discrepancy of order $n^{1 / 4}$. We investigate the discrepancy for the set system $\mathcal{S}_{n}^{3}$ formed by all sums of three arithmetic progressions on $[n]$ and show that the discrepancy of $\mathcal{S}_{n}^{3}$ is bounded below by $\Omega\left(n^{1 / 2}\right)$. Thus $\mathcal{S}_{n}^{3}$ is one of the few explicit examples of systems with polynomially many sets and a discrepancy this high.
\end{abstract}

\section{Introduction}

Let $(X, \mathcal{F})$ be a set system on a finite set. The discrepancy problem is to color each point of $X$ either red or blue, in such a way that any of the sets of $\mathcal{F}$ has roughly the same number of red points and blue points. The maximum deviation from an even splitting, over all sets of $\mathcal{F}$, is the discrepancy of $\mathcal{F}$, denoted by $\operatorname{disc}(\mathcal{F})$. Formally

$$
\operatorname{disc}(\mathcal{F})=\min _{\chi: X \rightarrow\{-1,1\}} \max _{S \in \mathcal{F}}\left|\sum_{x \in S} \chi(x)\right| .
$$

For further information see Beck and Sós [BS95], Chazelle [Cha00], and Matoušek [Mat99].

Let $n$ be a positive integer and let $[n]$ denote the set $\{0,1, \ldots, n-1\}$. For any $a \in \mathbf{Z}$ and $d_{1}, n_{1} \in \mathbf{N}$ we define the arithmetic progression $A P\left(a, d_{1}, n_{1}\right)$ as the set $\left\{a+i d_{1}: i \in\left[n_{1}\right]\right\}$. The set system formed by all arithmetic progressions on $[n]$ we denote by $\left([n], \mathcal{S}_{n}\right)$ where $\mathcal{S}_{n}=\left\{A P\left(a, d_{1}, n_{1}\right) \cap[n]: a, d_{1}, n_{1} \in \mathbf{N}\right\}$.

The lower bound $\Omega\left(n^{1 / 4}\right)$ on the discrepancy of arithmetic progressions $\mathcal{S}_{n}$ proved by Roth [Rot64] was one of the early results in combinatorial discrepancy. In 1974, Sárkőzy (see [ES74]) established an $O\left(n^{1 / 3+\epsilon}\right)$ upper bound. This was improved by Beck [Bec81],

\footnotetext{
*Supported by Czech Science Foundation grant 201/05/H014.
} 
who obtained the near-tight upper bound of $O\left(n^{1 / 4} \log ^{5 / 2} n\right)$, inventing the powerful partial coloring method for that purpose. The asymptotically tight upper bound $O\left(n^{1 / 4}\right)$ was finally proved by Matoušek and Spencer [MS96].

Discrepancies of related set systems were also studied. One possible extension of the original problem is to consider set systems formed by sums of arithmetic progressions, where a sum of $k$ arithmetic progressions $A P_{k}\left(a, d_{1}, \ldots, d_{k}, n_{1}, \ldots, n_{k}\right)$ is defined for $a \in \mathbf{Z}$ and $d_{1}, \ldots, d_{k}, n_{1}, \ldots, n_{k} \in \mathbf{N}$ as the set $\left\{a+i_{1} d_{1}+\ldots+i_{k} d_{k}: i_{l} \in\left[n_{l}\right], l=1, \ldots, k\right\}$. The corresponding set system of all sums of $k$ arithmetic progressions on $[n]$ is then $\left([n], \mathcal{S}_{n}^{k}\right)$, where $\mathcal{S}_{n}^{k}=\left\{A P_{k}\left(a, d_{1}, \ldots, d_{k}, n_{1}, \ldots, n_{k}\right) \cap[n]: a \in \mathbf{Z} ; d_{1}, \ldots, d_{k}, n_{1}, \ldots, n_{k} \in\right.$ $\mathbf{N}$ \}. Hebbinghaus [Heb04] proved that $\operatorname{disc}\left(\mathcal{S}_{n}^{k}\right)=\Omega\left(n^{\frac{k}{2 k+2}}\right)$. Here we show that for $k \geq 3, \operatorname{disc}\left(\mathcal{S}_{n}^{k}\right)=\Omega\left(n^{1 / 2}\right)$. Thus $\mathcal{S}_{n}^{3}$ is one of the few explicit examples of systems with polynomially many sets and a discrepancy this high.

For a fixed $k \geq 3$, the lower bound on $\mathcal{S}_{n}^{k}$ is nearly tight since the random coloring lemma [AS92] provides the upper bound $O\left(n^{1 / 2} \log ^{1 / 2} n\right)$. In the case $k=2$, there is still a considerable gap, $\Omega\left(n^{1 / 3}\right)$ versus $O\left(n^{1 / 2} \log ^{1 / 2} n\right)$, and estimating the correct bound remains still open.

We start in Section 2 with recalling the eigenvalue bound method and then we show how it can be used for wrapped set systems. In Section 3 we discuss how to construct suitable wrapped set systems and illustrate this approach on the system of arithmetic progressions on $[n]$ (this version of proof is attributed to Lovász). Then we construct a wrapped set system for our main result.

\section{Preliminaries}

In this section we recall some basic facts. We start with some definitions from discrepancy theory; for more definitions see [Mat99].

Let $(X, \mathcal{F})$ be a set system on a finite set. Let us enumerate the elements of $X$ as $x_{1}, x_{2}, \ldots, x_{n}$ and the sets of $\mathcal{F}$ as $S_{1}, S_{2}, \ldots, S_{m}$ in some arbitrary order. The incidence matrix of $(X, \mathcal{F})$ is the $m \times n$ matrix $A$, with columns corresponding to points of $X$ and rows corresponding to sets of $\mathcal{F}$, whose element $a_{i j}$ is given by

$$
a_{i j}= \begin{cases}1 & \text { if } j \in S_{i} \\ 0 & \text { otherwise. }\end{cases}
$$

As we will see, it is useful to reformulate the definition of the discrepancy of $\mathcal{F}$ in terms of the incidence matrix. Now let us regard a coloring $\chi: X \rightarrow\{-1,+1\}$ as the column vector $\left(\chi\left(x_{1}\right), \chi\left(x_{2}\right), \ldots, \chi\left(x_{n}\right)\right)^{T} \in \mathbf{R}^{n}$. Then the product $A \chi$ is the row vector $\left(\chi\left(S_{1}\right), \chi\left(S_{2}\right), \ldots, \chi\left(S_{n}\right)\right) \in \mathbf{R}^{m}$, where we extend the coloring $\chi$ for sets as $\chi(S)=$ $\sum_{x \in S} \chi(x)$. Therefore, the definition of the discrepancy of $\mathcal{F}$ can be written as

$$
\operatorname{disc}(\mathcal{F})=\min _{x \in\{-1,1\}^{n}}\|A x\|_{\infty} .
$$


For many lower bound techniques, it is easier to consider the $L_{2}$-discrepancy instead of the worst-case discrepancy. In our case, this means replacing the max-norm $\|\cdot\|_{\infty}$ by the usual Euclidean norm $\|$.$\| . Namely, we have$

$$
\operatorname{disc}(\mathcal{F}) \geq \operatorname{disc}_{2}(\mathcal{F})=\min _{\chi}\left(\frac{1}{m} \sum_{i=1}^{m} \chi\left(S_{i}\right)^{2}\right)^{1 / 2}=\frac{1}{\sqrt{m}} \cdot \min _{x \in\{-1,1\}^{n}}\|A x\| .
$$

To obtain a lower bound on the $L_{2}$-discrepancy for a set system, we can use the following eigenvalue lower bound:

Theorem 2.1 (Eigenvalue bound, see [BS95]) Let $(X, \mathcal{F})$ be a system of $m$ sets on an n-point set, and let $A$ denote its incidence matrix. Then we have

$$
\operatorname{disc}(\mathcal{F}) \geq \operatorname{disc}_{2}(\mathcal{F}) \geq \sqrt{\frac{n}{m} \cdot \lambda_{\text {min }}},
$$

where $\lambda_{\min }$ denotes the smallest eigenvalue of the $n \times n$ matrix $A^{T} A$.

The computation of eigenvalues becomes much easier when the matrix $A^{T} A$ is a circulant matrix. A circulant matrix is an $n \times n$ matrix whose rows are composed of cyclically shifted copies of the first row. Namely, for an $n$-dimensional vector $\left(a_{0}, a_{1}, \ldots, a_{n-1}\right)$ we define the $n \times n$ circulant matrix $C\left(a_{0}, a_{1}, \ldots, a_{n-1}\right)$ by putting $c_{i j}=a_{(j-i) \bmod n}$, i.e.

$$
C\left(a_{0}, a_{1}, \ldots, a_{n-1}\right)=\left(\begin{array}{ccccc}
a_{0} & a_{1} & a_{2} & \ldots & a_{n-1} \\
a_{1} & a_{2} & a_{3} & \ldots & a_{0} \\
a_{2} & a_{3} & a_{4} & \ldots & a_{1} \\
\cdot & \cdot & \cdot & \cdot & \cdot \\
a_{n-1} & a_{0} & a_{1} & \ldots & a_{n-2}
\end{array}\right) .
$$

Let $\zeta_{0}, \zeta_{1}, \ldots, \zeta_{n-1}$ denote the $n$-th roots of the unity, which are defined as roots of the cyclotomic equation $x^{n}=1$. All the roots lie on the unit circle and we can order them according to the sequence of visiting them if we go around the unit circle counterclockwise starting at 0 , namely we put $\zeta_{k}=e^{\frac{2 \pi i}{n} k}$. This simplifies the following operations:

- $\zeta_{j} \zeta_{k}=\zeta_{(j+k) \bmod n}$

- $\zeta_{j}^{k}=\zeta_{(j k) \bmod n}$

For convenience, we will consider all operations + , . on indices reduced modulo $n$ and thus we will later omit the $\bmod n$ suffix.

We define the complex argument as usual by $\arg (x+i y)=\arctan \left(\frac{y}{x}\right)$ and restrict its range to the interval $(-\pi,+\pi]$. The complex argument of the $n$-th root of unity is then as follows

$$
\arg \left(\zeta_{k}\right)= \begin{cases}\frac{2 \pi k}{n} & \text { if } 0 \leq k \leq \frac{n}{2} \\ \frac{2 \pi(k-n)}{n} & \text { if } \frac{n}{2}<k<n .\end{cases}
$$


Let $B$ be a circulant matrix $C\left(a_{0}, a_{1}, \ldots, a_{n-1}\right)$. It can be easily verified that $z_{i}=$ $\left(1, \zeta_{i}^{1}, \zeta_{i}^{2}, \ldots, \zeta_{i}^{n-1}\right)^{T}$ is an eigenvector of $B$ and thus the eigenvalues $\lambda_{0}, \lambda_{1}, \ldots, \lambda_{n-1}$ of $B$ are

$$
\lambda_{i}=a_{0}+a_{1} \zeta_{i}+a_{2} \zeta_{i}^{2}+\ldots+a_{n-1} \zeta_{i}^{n-1}
$$

Let $A$ be an incidence matrix for the set system $(X, \mathcal{F})$ and let $B$ denote $A^{T} A$. Then the element $b_{i j}$ counts the number of sets $S_{i} \in \mathcal{F}$ containing both elements $x_{i}$ and $x_{j}$. Moreover, if the matrix $B$ is a circulant matrix $C\left(a_{0}, a_{1}, \ldots, a_{n-1}\right)$, we can derive a more useful expression for the eigenvalues of $B$ :

$$
\begin{aligned}
n \lambda_{k} & =n \sum_{i=0}^{n-1} a_{i} \zeta_{k}^{i}=\sum_{i=0}^{n-1} \sum_{j=0}^{n-1} a_{(i-j) \bmod n} \zeta_{k}^{(i-j) \bmod n}=\sum_{i=0}^{n-1} \sum_{j=0}^{n-1} b_{i j} \zeta_{k}^{(i-j)}= \\
& =\sum_{S \in \mathcal{F}} \sum_{x_{i} \in S} \sum_{x_{j} \in S} \zeta_{k}^{(i-j)}=\sum_{S \in \mathcal{F}}\left|\sum_{x_{i} \in S} \zeta_{k}^{i}\right|^{2} .
\end{aligned}
$$

And thus

$$
\lambda_{k}=\frac{1}{n} \sum_{S \in \mathcal{F}}\left|\sum_{x_{i} \in S} \zeta_{k}^{i}\right|^{2} .
$$

Let $(X, \mathcal{F})$ be a set system, where $X=[n]$ and $\mathcal{F}$ contains exactly $m n$ sets enumerated as $\mathcal{F}=\left\{S_{0}, S_{1}, \ldots, S_{m n-1}\right\}$. We say that a set system $(X, \mathcal{F})$ is wrapped if for every $i \in[m]$ and $j \in[n]$ the set $S_{i n+j}$ is the set $S_{i n}$ cyclically translated by $j$, i.e.

$$
S_{i n+j}=\left\{(k+j) \bmod n: k \in S_{i}\right\} .
$$

The incidence matrix $A$ of a wrapped set system $(X, \mathcal{F})$ is composed of $m$ square $n \times n$ circulant matrices $A_{0}, A_{1}, \ldots, A_{m-1}$ stacked up vertically, one on top of the other:

$$
A=\left(\begin{array}{c}
A_{0} \\
A_{1} \\
\vdots \\
A_{m-1}
\end{array}\right) .
$$

By the definition of a wrapped set system every $A_{i}$ is a circulant and thus every $A_{i}^{T} A_{i}$ is a circulant too. Note that although $A$ is not a circulant itself, the matrix $B=A^{T} A$ is equal to $\sum_{i=0}^{m-1} A_{i}^{T} A_{i}$ and therefore $B$ is a circulant.

Alternatively, we can observe that the $(i, j)$ entry of $A^{T} A$ is the number of sets from $\mathcal{F}$ that contain both elements $i$ and $j$. Since the sets forming $\mathcal{F}$ are invariant under cyclic shifts, the entries $(i, j)$ and $(i+k, j+k)$ of $A^{T} A$ are the same for an arbitrary shift by $k$ and thus $A^{T} A$ is a circulant. 
Lemma 2.2 Let $(X, \mathcal{F})$ be a wrapped set system, where $|X|=n$ and $|\mathcal{F}|=m n$, and let $A$ be its incidence matrix. Then the $n \times n$ matrix $B=A^{T} A$ is a circulant and its eigenvalues are

$$
\lambda_{k}=\sum_{i=0}^{m-1}\left|\sum_{j \in S_{i n}} \zeta_{k}^{j}\right|^{2} .
$$

Proof. Since for each set $S_{\text {in }}$, we have its $n-1$ translates in $\mathcal{F}$ that give the same contribution, we may just count $n$-times the contribution of the set $S_{i n}$ and thus

$$
\lambda_{j}=\frac{1}{n} \sum_{S \in \mathcal{F}}\left|\sum_{k \in S} \zeta_{j}^{k}\right|^{2}=\sum_{i=0}^{m-1}\left|\sum_{k \in S_{i n}} \zeta_{j}^{k}\right|^{2} .
$$

\section{Lower bounds}

In this section we will prove the lower bound for the sums of three arithmetic progressions. For this purpose we will use following lemma:

Lemma 3.1 Let $([n], \mathcal{F})$ be a wrapped set system, where $|\mathcal{F}|=m n$, and let $\zeta_{0}, \zeta_{1}, \ldots, \zeta_{n-1}$ be the $n$-th roots of unity. If there are real constants $c, \alpha>0$ such that for each $j \in[n]$ there is $S_{\text {in }} \in \mathcal{F}$ such that

$$
\left|\sum_{k \in S_{i n}} \zeta_{j}^{k}\right| \geq c n^{\alpha}
$$

holds, then $\operatorname{disc}(\mathcal{F}) \geq \frac{c n^{\alpha}}{\sqrt{m}}$.

Proof. To invoke the eigenvalue bound for an $L_{2}$-discrepancy we need to lowerbound the value of smallest eigenvalue $\lambda_{\min }$. Since our set system is wrapped, we know that all eigenvalues are given by the expression

$$
\lambda_{j}=\sum_{i=0}^{m-1}\left|\sum_{k \in S_{i n}} \zeta_{j}^{k}\right|^{2}
$$

We know that for each $j$ there is a set $S_{i n}$ that makes the eigenvalue 'large' and hence for every eigenvalue we know that

$$
\lambda_{j}=\sum_{i=0}^{m-1}\left|\sum_{k \in S_{i n}} \zeta_{j}^{k}\right|^{2} \geq\left|\sum_{k \in S_{i n}} \zeta_{j}^{k}\right|^{2} \geq c^{2} n^{2 \alpha} .
$$

Thus

$$
\operatorname{disc}(\mathcal{F}) \geq \operatorname{disc}_{2}(\mathcal{F}) \geq \sqrt{\frac{n}{m n} c^{2} n^{2 \alpha}}=\frac{c n^{\alpha}}{\sqrt{m}} .
$$


If we want to obtain a good lower bound from Lemma 3.1, the number of sets forming $\mathcal{F}$ has to be small and $\mathcal{F}$ has to contain for each $j \in[n]$ a set $B_{j}$, such that $\left|\sum_{k \in B_{j}} \zeta_{j}^{k}\right|$ is large. Our goal is to ensure that for each $j \in[n]$ there is a $B_{j} \in \mathcal{F}$ such that all $\zeta_{j}^{k}$ for $k \in B_{j}$ are concentrated in one part of the unit circle. Namely, if all $k \in B_{j}$ satisfy

$$
-\frac{\pi}{3} \leq \arg \zeta_{j}^{k} \leq \frac{\pi}{3}
$$

then $\operatorname{Re} \zeta_{j}^{k} \geq \frac{1}{2}$ for all $k \in B_{j}$, and the value of $\left|\sum_{k \in B_{j}} \zeta_{j}^{k}\right|$ will be at least $\left|B_{j}\right| / 2$.

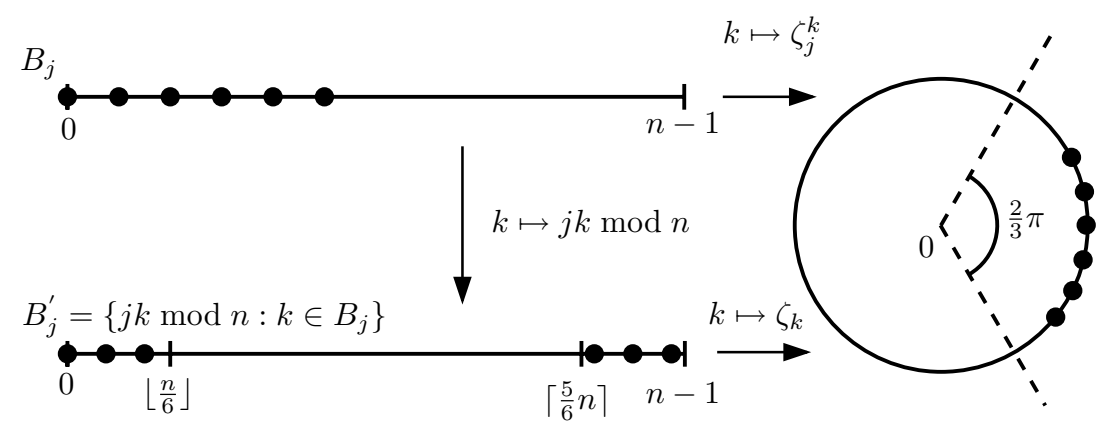

Figure 1: The relation of the set $B_{j}^{\prime}$ and the $\operatorname{sum} \sum_{k \in B_{j}} \zeta_{j}^{k}$

For convenience, we define for every $B_{j} \subseteq[n]$ a set $B_{j}^{\prime}$ as the set $\left\{j k \bmod n: k \in B_{j}\right\}$. The set $B_{j}^{\prime}$ is actually the set of indices $i$ of $\zeta_{i}=\zeta_{j}^{k}$ that participate in the sum $\sum_{k \in B_{j}} \zeta_{j}^{k}$ (see figure 1). The condition $\left|\arg \zeta_{k}\right| \leq \frac{\pi}{3}$ for all $k \in B_{j}$ is thus equivalent to the condition

$$
B_{j}^{\prime} \subseteq\left\{0, \ldots,\left\lfloor\frac{1}{6} n\right\rfloor\right\} \cup\left\{\left\lceil\frac{5}{6} n\right\rceil, \ldots, n-1\right\} .
$$

Moreover, if $n$ is a prime and $0<j<n$, the mapping $k \mapsto j k \bmod n$ is a bijection and the cardinalities of $B_{j}$ and $B_{j}^{\prime}$ are the same.

Now let us apply this method to prove the $\Omega\left(n^{1 / 4}\right)$ lower bound for the set system of arithmetic progressions on $[n]$. For this purpose we construct a small auxiliary wrapped set system $\mathcal{F}$ that is suitable for Lemma 3.1 and $\operatorname{disc}\left(\mathcal{S}_{n}\right)$ is asymptotically bounded below by $\operatorname{disc}(\mathcal{F})$.

We will show, that for each $j \in[n]$ we can find a positive integer $d_{j}=O(\sqrt{n})$, such that $\left|\arg \zeta_{j}^{d_{j}}\right|=O\left(n^{-1 / 2}\right)$. Let us take as $B_{j}$ an arithmetic progression with difference $d_{j}$ having $\Omega(\sqrt{n})$ elements, such that $\left|\arg \zeta_{j}^{k}\right| \leq \frac{\pi}{3}$ for all $k \in B_{j}$. For such a $B_{j}$, we get $\left|\sum_{k \in B_{j}} \zeta_{j}^{k}\right|=\Omega(\sqrt{n})$. Since there are only $O(\sqrt{n})$ possible choices of $d_{j}$, it suffices us to put only $O(\sqrt{n})$ different sets $B_{j}$ into $\mathcal{F}$. With each inserted $B_{j}$, we also have to put into $\mathcal{F}$ its $n-1$ wrapped translates, and thus $\mathcal{F}$ has size $O\left(n^{3 / 2}\right)$. The following theorem 
summarizes our discussion. This version of the proof of the lower bound for $\mathcal{S}_{n}$ was first suggested by Lovász and can be found in [BS95].

Theorem 3.2 For any $n \in \mathbf{N}$ we put $k=\lfloor\sqrt{n / 6}\rfloor$ and $m=6 k$. Let us consider the following set system $([n], \mathcal{F})$, where $\mathcal{F}=\left\{S_{0}, S_{1}, \ldots, S_{m n-1}\right\}$ and the sets $S_{d n+j}$ for $d \in[m]$ and $j \in[n]$ are given as

$$
S_{d n+j}=\{(d i+j) \bmod n: i \in[k]\} .
$$

Then

$$
\operatorname{disc}(\mathcal{F}) \geq c n^{1 / 4}
$$

Proof. For a fixed $j \in[n]$, there is by the Pigeonhole Principle a positive integer $c_{0}$, $1 \leq c_{0} \leq m$ such that

$$
-\frac{2 \pi}{m} \leq \arg \left(\zeta_{j}^{c_{0}}\right) \leq \frac{2 \pi}{m}
$$

Then $\operatorname{Re} \zeta_{j}^{i c_{0}} \geq 1 / 2$ for $0 \leq i \leq k-1$, and hence

$$
\left|\sum_{i \in S_{c_{0} n}} \zeta_{j}^{i}\right| \geq\left(\operatorname{Re} \sum_{i \in S_{c_{0} n}} \zeta_{j}^{i}\right) \geq k / 2 .
$$

From this and Lemma 3.1 it immediately follows that

$$
\operatorname{disc}(\mathcal{F})>\frac{1}{10} n^{1 / 4}
$$

Since every $S \in \mathcal{F}$ from theorem 3.2 is a disjoint union of two arithmetic progressions, we get the following corollary.

Corollary 3.3 For $n \in \mathbf{N}$, let $\left([n], \mathcal{S}_{n}\right)$ be a set system formed by all arithmetic progressions on $[n]$. Then $\operatorname{disc}\left(\mathcal{S}_{n}\right)=\Omega\left(n^{1 / 4}\right)$.

For the set system $\left([n], \mathcal{S}_{n}\right)$ is the $\Omega\left(n^{1 / 4}\right)$ lower bound tight. We would like to show that the set systems $\left([n], \mathcal{S}_{n}^{k}\right)$ for $k \geq 2$ have their discrepancy bounded below by $\Omega\left(n^{1 / 2}\right)$. Unfortunately, we are able to prove this only for $k \geq 3$, while for $k=2$ the currently known best lower bound is $\Omega\left(n^{1 / 3}\right)$; see [Heb04].

As we have seen in the proof of Theorem 3.2, for each $j \in\{1, \ldots, n-1\}$ we can find two positive integers $0<c_{1} \leq \sqrt{n}$ and $0<d_{1} \leq \sqrt{n}$, such that $\left|\arg \zeta_{j}^{c_{1}}\right|=\frac{2 \pi d_{1}}{n}$. Without loss of generality, let us assume that $\arg \zeta_{j}^{c_{1}}$ is positive and thus $d_{1}=c_{1} j \bmod n$, the other case can be handled in the same way. Let $A_{j}$ be the set $\left\{i c_{1}: i \in\left[n_{1}\right]\right\}$. If $n_{1} \leq \min \left\{\frac{n}{c_{1}}, \frac{n}{6 d_{1}}\right\}$, then $A_{j}$ is an arithmetic progression on $[n]$ and $A_{j}^{\prime}$ is an arithmetic progression on $[\lfloor n / 6\rfloor]$ (see figure 2). Although is $n_{1}$ is at least $\Omega(\sqrt{n})$, we cannot generally expect a greater value. 


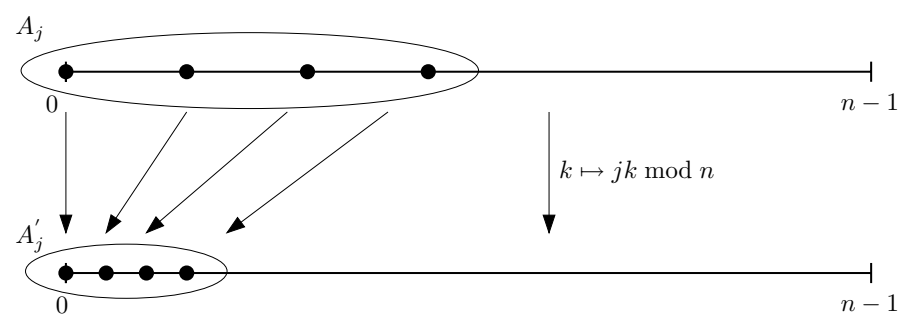

Figure 2: $A_{j}$ with difference $c_{1}$ goes to $A_{j}^{\prime}$ with difference $d_{1}$

Our goal is to find a $B_{j}$ such that $B_{j}^{\prime}$ covers a constant fraction of $\left\{0, \ldots,\left\lfloor\frac{1}{6} n\right\rfloor\right\} \cup$ $\left\{\left\lceil\frac{5}{6} n\right\rceil, \ldots, n-1\right\}$. In next two steps we will schematically (and possibly misleadingly) show how to achieve this. In the first step we extend the arithmetic progression $A_{j}^{\prime}$ to a longer arithmetic progression $B_{j}^{\prime}$ with the same difference. This is done in such a way that $B_{j}^{\prime}$ consists of several copies of $A_{j}^{\prime}$ and thus $B_{j}$ is taken as a sum of two arithmetic progressions (see figure 3 ). In this way we can have $\Omega\left(n / d_{1}\right)$ elements in $B_{j}$.

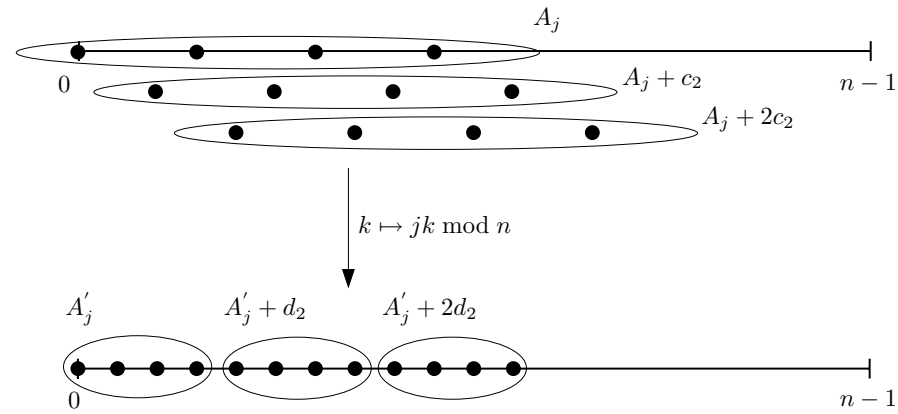

Figure 3: $B_{j}\left(\right.$ resp. $\left.B_{j}^{\prime}\right)$ composed from copies of $A_{j}\left(\right.$ resp. $\left.A_{j}^{\prime}\right)$

In the last step we take a suitable sum of three arithmetic progressions for $C_{j}$ such that $C_{j}^{\prime}$ is composed of $\Omega\left(d_{1}\right)$ interlaced copies of $B_{j}^{\prime}$ that are mutually disjoint (see figure $4)$, and thus $C_{j}^{\prime}$ has $\Omega(n)$ elements.

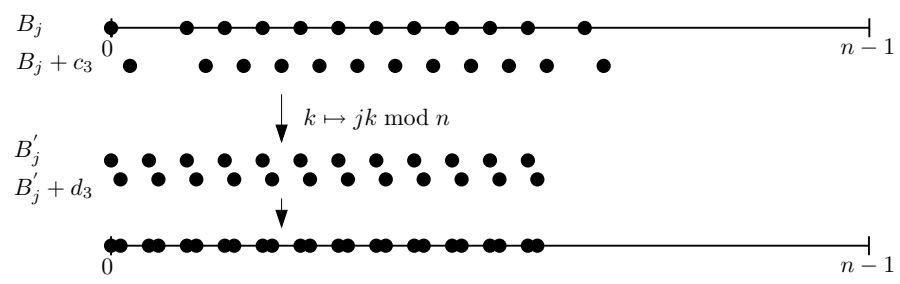

Figure 4: $C_{j}^{\prime}$ is composed from interlaced copies of $B_{j}^{\prime}$ 
The following lemma provides, for each $j \in\{1, \ldots, n-1\}$, a precise and more careful construction of the set $C_{j}$. This construction requires $n$ to be a prime.

Lemma 3.4 Let $n$ be a prime. For each $j \in\{1 \ldots n-1\}$ there exists a set $C_{j}$ such that

- $C_{j}$ is a sum of three arithmetic progressions on $[n]$

- $\operatorname{Re} \zeta_{j}^{k} \geq 1 / 2$ for every $k \in C_{j}$

- $\left|C_{j}\right| \geq \frac{1}{5000} n$.

Proof. For a fixed $j$ we find integer constants $c_{1}, c_{2}, c_{3}, d_{1}, d_{2}, d_{3}, n_{1}, n_{2}$ and $n_{3}$ as follows:

1. Let $c_{1}$ be the $k \in\{1 \ldots\lfloor\sqrt{n}\rfloor\}$ for which the value $\operatorname{Re} \zeta_{j}^{k}$ is maximum. We put $d_{1}=\min \left\{j c_{1} \bmod n,-j c_{1} \bmod n\right\}$ and $n_{1}=\left\lceil\frac{n}{12 \max \left\{c_{1}, d_{1}\right\}}\right\rceil$.

2. If $c_{1} \leq 12 d_{1}$, then we put $c_{2}=1, d_{2}=1$ and $n_{2}=1$, otherwise we put $c_{2}=n \bmod c_{1}$, $d_{2}=d_{1}\left\lceil\frac{n}{c_{1}}\right\rceil$ and $n_{2}=\left\lfloor\frac{c_{1}}{30 d_{1}}\right\rfloor$.

3. If $d_{1}<6$, then we put $c_{3}=1, d_{3}=1$ and $n_{3}=1$, otherwise we put $c_{3}$ to be the $k \in\left\{1 \ldots\left\lfloor\frac{2 n}{d_{1}}\right\rfloor\right\}$ for which the value $\operatorname{Re} \zeta_{j}^{k}$ is maximum. We put $d_{3}=$ $\min \left\{j c_{3} \bmod n,-j c_{3} \bmod n\right\}$ and $n_{3}=\left\lfloor\frac{d_{1}}{12}\right\rfloor$.

4. We put $C_{j}=\left\{i_{1} c_{1}+i_{2} c_{2}+i_{3} c_{3}: i_{k} \in\left[n_{k}\right], k=1,2,3\right\}$.

We have chosen $c_{1}$ as the $k \in\{1 \ldots\lfloor\sqrt{n}\rfloor\}$ for which the value of $\operatorname{Re} \zeta_{j}^{k}$ is maximum, i.e. as the $k \in\{1 \ldots\lfloor\sqrt{n}\rfloor\}$ for which the value of $\left|\arg \zeta_{j}^{k}\right|$ is minimum. By the Pigeonhole Principle

$$
-\frac{2 \pi}{\lceil\sqrt{n}\rceil} \leq \arg \zeta_{j}^{c_{1}} \leq \frac{2 \pi}{\lceil\sqrt{n}\rceil},
$$

and since $\left|\arg \zeta_{j}^{c_{1}}\right|=\arg \zeta_{d_{1}}=\frac{2 \pi d_{1}}{n}$, we conclude that $d_{1} \leq \sqrt{n}$. Similarly we arrive at $c_{3} \leq \frac{2 n}{d_{1}}$ and $d_{3} \leq \frac{d_{1}}{2}$

Claim A: $C_{j}$ is a sum of three arithmetic progressions on $[n]$.

By construction the set $C_{j}$ is a sum of three arithmetic progressions. The largest element of $C_{j}$ is bounded by

$$
\begin{aligned}
\max C_{j} & =c_{1}\left(n_{1}-1\right)+c_{2}\left(n_{2}-1\right)+c_{3}\left(n_{3}-1\right) \leq \\
& \leq \frac{n}{12}+\frac{n}{30}+\frac{n}{6} \leq \frac{n}{2}
\end{aligned}
$$

and thus $C_{j} \subseteq[n]$. 
Claim B: $\operatorname{Re} \zeta_{j}^{k} \geq 1 / 2$ for every $k \in C_{j}$.

We show that for every $k \in C_{j}$ the value of $\left|\arg \zeta_{j}^{k}\right|$ is less than $\pi / 3$ and this already implies the claim.

$$
\begin{aligned}
\max _{k \in C_{j}}\left|\arg \zeta_{j}^{k}\right| & =\max _{\left(i_{1}, i_{2}, i_{3}\right) \in\left[n_{1}\right] \times\left[n_{2}\right] \times\left[n_{3}\right]}\left|\arg \zeta_{j}^{c_{1} i_{1}}+\arg \zeta_{j}^{c_{2} i_{2}}+\arg \zeta_{j}^{c_{3} i_{3}}\right| \leq \\
& \leq \max _{i_{1} \in\left[n_{1}\right]}\left|\arg \zeta_{j}^{c_{1} i_{1}}\right|+\max _{i_{2} \in\left[n_{2}\right]}\left|\arg \zeta_{j}^{c_{2} i_{2}}\right|+\max _{i_{3} \in\left[n_{3}\right]}\left|\arg \zeta_{j}^{c_{3} i_{3}}\right|= \\
& =\frac{2 \pi}{n}\left(d_{1}\left(n_{1}-1\right)+d_{2}\left(n_{2}-1\right)+d_{3}\left(n_{3}-1\right)\right) .
\end{aligned}
$$

In the case that $c_{1} \leq 12 d_{1}$

$$
\begin{aligned}
\max _{k \in C_{j}}\left|\arg \zeta_{j}^{k}\right| & =\frac{2 \pi}{n}\left(d_{1}\left(n_{1}-1\right)+d_{2}\left(n_{2}-1\right)+d_{3}\left(n_{3}-1\right)\right) \leq \\
& \leq \frac{2 \pi}{n}\left(d_{1} \frac{n}{12 d_{1}}+d_{2} \cdot 0+\frac{d_{1}}{2} \frac{d_{1}}{12}\right) \leq \frac{\pi}{3},
\end{aligned}
$$

otherwise

$$
\begin{aligned}
\max _{k \in C_{j}}\left|\arg \zeta_{j}^{k}\right| & =\frac{2 \pi}{n}\left(d_{1}\left(n_{1}-1\right)+d_{2}\left(n_{2}-1\right)+d_{3}\left(n_{3}-1\right)\right) \leq \\
& \leq \frac{2 \pi}{n}\left(d_{1} \frac{n}{144 d_{1}}+d_{1}\left\lceil\frac{n}{c_{1}}\right\rceil \frac{c_{1}}{30 d_{1}}+\frac{d_{1}}{2} \frac{d_{1}}{12}\right) \leq \frac{\pi}{3}
\end{aligned}
$$

Claim C: $\left|C_{j}\right| \geq \frac{1}{5000} n$.

We put $D=\left\{i_{1} d_{1}+i_{2} d_{2}: i_{1} \in\left[n_{1}\right], i_{2} \in\left[n_{2}\right]\right\}$. From the fact that $d_{2}=d_{1}\left\lceil\frac{n}{c_{1}}\right\rceil$ and $d_{2}>n_{1} d_{1}$ we deduce that $D$ is a subset of an arithmetic progression with difference $d_{1}$ and $|D|=n_{1} n_{2}$.

The set $E=\left\{i_{1} d_{1}+i_{2} d_{2}+i_{3} d_{3}: i_{1} \in\left[n_{1}\right], i_{2} \in\left[n_{2}\right], i_{3} \in\left[n_{3}\right]\right\}$ is a union of $n_{3}$ shifted copies of $D$. Our goal is to show that those $n_{3}$ shifted copies of $D$ are mutually disjoint. If there were two intersecting copies of $D$, then there has to exist a $k \in\left\{1, \ldots, n_{3}\right\}$, such that $d_{1} \mid k d_{3}$. Let it be so and let $k, l$ be the integers demonstrating this case, i.e. $l d_{1}=k d_{3}$. Since $d_{1}>d_{3}$, we have $l<k$. Thus $l<n_{3} \leq n_{1}$ and the preimage of $l d_{1}$ under the mapping $f_{j}(k)=j k \bmod n$ is $f_{j}^{-1}\left(l d_{1}\right)=l c_{1}$ and similarly $f_{j}^{-1}\left(k d_{3}\right)=k c_{3}$. The mapping $f_{j}$ is a bijection and therefore $l d_{1}=k d_{3}$ implies $l c_{1}=f_{j}^{-1}\left(l d_{1}\right)=f_{j}^{-1}\left(k d_{3}\right)=k c_{3}$. But since we also have $c_{1}<c_{3}$, we arrive at the contradiction $l>k$. Thus all $n_{3}$ shifted copies of $D$ are mutually disjoint and $\left|C_{j}\right|=|E|=n_{1} n_{2} n_{3} \geq n / 5000$.

Theorem 3.5 For each prime $n$ there exists a wrapped set system $\left([n], \mathcal{F}_{n}\right)$, where $\mathcal{F}_{n}=$ $\left\{S_{0}, S_{1}, \ldots, S_{n^{2}-1}\right\}$ such that each $S_{i} \in \mathcal{F}_{n}$ is a union of two sums of three arithmetic progressions and

$$
\operatorname{disc}\left(\mathcal{F}_{n}\right)>\frac{1}{10000} n^{1 / 2}
$$


Proof. For a fixed $n$ we construct $\mathcal{F}_{n}=\left\{S_{0}, S_{1}, \ldots, S_{n^{2}-1}\right\}$ as follows: For $S_{0}$ just take the set $\{0,1, \ldots\lfloor n / 5000\rfloor\}$ and for $0<j<n$ we put $S_{j n}=C_{j}$ as constructed in Lemma 3.4. Since for all $0 \leq j<n$ we know that

$$
\left|\sum_{k \in S_{j n}} \zeta_{j}^{k}\right| \geq \sum_{k \in S_{j n}} \operatorname{Re} \zeta_{j}^{k} \geq \frac{1}{10000} n,
$$

from lemma 3.1 it immediately follows that

$$
\operatorname{disc}(\mathcal{S})>\frac{1}{10000} n^{1 / 2}
$$

Corollary 3.6 For $n \in \mathbf{N}$, let $\left([n], \mathcal{S}_{n}\right)$ be a set system formed by all sums of three arithmetic progressions on $[n]$. Then $\operatorname{disc}\left(\mathcal{S}_{n}\right)=\Omega\left(n^{1 / 2}\right)$.

\section{Acknowledgements}

I would like to thank Jiří Matoušek for introducing me into the area and for help with writing this paper, and Lisa Bailey for reading the paper and correcting grammatical errors.

\section{References}

[AS92] N. Alon and J. Spencer. The Probabilistic Method. J. Wiley and Sons, New York, NY, 1992.

[Bec81] J. Beck. Roth's Estimate of the Discrepancy of Integer Sequences is Nearly Sharp. Combinatorica 1(4):319-325, 1981.

[BS95] J. Beck and V. Sós. Discrepancy Theory. In Handbook of Combinatorics, pages 1405-1446. North-Holland, Amsterdam, 1995.

[Cha00] B. Chazelle. The Discrepancy Method. Cambridge University Press, 2000.

[ES74] P. Erdős and J. Spencer. Probabilistic Methods in Combinatorics. Academic Press, 1974.

[Heb04] N. Hebbinghaus. Discrepancy of Sums of Arithmetic Progressions. In Electronic Notes in Discrete Mathematics 17C, pages 185-189, 2004.

[Mat99] J. Matoušek. Geometric Discrepancy. Springer, 1999.

[MS96] J. Matoušek and J.Spencer. Discrepancy in Arithmetic Progressions. Journal of the American Mathematic Society, 9:195-204, 1996.

[Rot64] K. F. Roth. Remark Concerning Integer Sequences. Acta Arithmetica 9:257-260, 1964. 